\title{
Protocol for the management of oral surgery patients on warfarin utilizing a Point-of-Care In-Office international normalized ratio monitoring device
}

\author{
Gregory P. Hatzis \\ Center for Facial, Oral \& Implant Surgery, Good Shepherd Medical Center, Longview, USA \\ Email: gregoryhatzis@me.com \\ Received 11 March 2013; revised 12 April 2013; accepted 17 May 2013 \\ Copyright (C) 2013 Gregory P. Hatzis. This is an open access article distributed under the Creative Commons Attribution License, \\ which permits unrestricted use, distribution, and reproduction in any medium, provided the original work is properly cited.
}

\begin{abstract}
Purpose: This study was performed to assess the utility and safety of an In-Office INR Monitoring Device and present a safe and efficient protocol for the management of patients on oral anticoagulants and/or antithrombolytics requiring routine office oral and maxillofacial surgery. Patients and Methods: Sixtyone patients requiring "minor" oral and maxillofacial surgery being treated chronically with oral anticoagulation (warfarin) were entered into the study and compared in 2 groups. The control group $(n=29)$ was managed by discontinuing warfarin and any antiplatelet medication(s) prior to surgery. In the study group $(n=30)$, the decision to continue or withhold warfarin was determined by a protocol in which patients are 1) stratified based on risk for thromboembolism, and 2) classified as requiring "major" or "minor" surgery. Procedures categorized as "minor" surgery included dental extraction(s), dental implants, soft tissue and bone biopsies, and pre-prosthetic bone surgery, and incision and drainage. Warfarin and antiplatelet medication were not withheld in these patients, and a Point-of-Care In-Office INR Monitoring Device was used to obtain INR levels on the day of consultation and surgery. Local measures including removal of granulation tissue, packing, suturing, etc. were utilized for hemostasis. Results: The 30 patients in the study group maintained on warfarin readily achieved hemostasis using intraoperative local measures. The mean INR measured by the In-Office INR Monitoring Device was 2.36 with a range from 1.3 to 3.2 . Study group patients underwent a total of 131 separate procedures including 108 dental extractions (impactions), placement of dental implants, pre-prosthetic bony surgery, bone cyst removal, soft tissue biopsies, facial skin cancer repair,
\end{abstract}

and incision and drainage. One patient (3\%) required "minor" intervention with removal of a "liver clot" on post-op day 2 with repacking and suturing. The 29 patients in the control group discontinued off of warfarin underwent a total of 99 procedures. One patient (3\%) also required a "minor" intervention (repacking of extraction site). There were no "major" complications in either group. Conclusions: This study supports previous studies that minor oral surgery procedures can be safely performed while maintaining patients on warfarin minimizing the risk of a potentially devastating thromboembolic event. When deciding whether or not to withhold warfarin, this study supports the use of the proposed protocol based on 1) risk stratification for thromboembolism, 2) the need for "minor" versus "major" surgery, 3) and utilization of an In-Office INR Monitoring Device. An In-Office Point-of-Care INR measuring device can be a very effective tool to safely simplify and make the perioperative management of the anticoagulated patient more efficient for the patient and oral and maxillo facial surgeon.

Keywords: Point-Of-Care; In-Office; INR; International Normalized Ratio; Thromboembolism; Perioperative Care; Anticoagulated; Oral and Maxillofacial Surgery; Extraction; Warfarin; Coumadin; Oral Surgery; Hemostasis; Local Hemostatic Measures; Antithrombolytics; Protocol; Packing; Suturing

\section{INTRODUCTION}

The perioperative management of the anticoagulated patient can be an inconvenient and cumbersome process for both the patient and surgeon, commonly involving direct communication with the treating physician, the discontinuation of warfarin, synchronized office visits, 
trips to the anticoagulation clinic, or even hospitalization. In recent years Point-of-Care devices to monitor International Normalized Ratio (INR) levels have become readily available and simple for patients to use at home. Similarly, oral and maxillofacialsurgeons may benefit from incorporating Point-of-Care coagulometers into the office to simplify the process of managing anticoagulated patients for both the patient and doctor.

When managing patients on anticoagulants, an individual assessment of risk for perioperative hemorrhagic must be weighed against the risks for thromboemolic events. The controversy [1,2] over the approach options when managing perioperative patients treated with oral anticoagulation may include one of the following three protocols:

1) continuing warfarin (Coumadin ${ }^{\circledR}$, Dupont Pharmaceuticals, Wilmington, Del.) therapy;

2) withholding warfarin therapy for a specified period of time before and/or after the procedure;

3) temporarily withholding warfarin therapy while a using a "heparin bridge".

Interruption of antithrombotic therapy exposes patients to an increased risk for thromboembolic events, such as stroke or mechanical valve thrombosis. These events can have devastating clinical consequences. The 2008 consensus guidelines from the American College of Chest Physicians Evidence-Based Clinical Practice Guidelines [3] recommend continuing warfarin in patients undergoing minor dental procedures around the time of the procedure and co-administering an oral pro-hemostatic agent (Grade 1B evidence). The Guidelines also recommend continuing aspirin (Grade $1 \mathrm{C}$ evidence). Similarly, in patients who are undergoing minor dermatological procedures the guidelines recommend continuing warfarin (Grade 1C evidence). Despite these recommendations, anticoagulants continue to be routinely discontinued.

The greatest problem encountered, as identified in surveys [4-6] is that no consensus exists regarding the optimal perioperative management of patients receiving anticoagulation undergoing minor oral surgery procedures. Traditionally, in order to obtain a suitable "window" for surgery, practitioners have relied on protocolswith preoperative holding of warfarin and hospital admission for administration of a heparin bridge [7,8]. The protocol then evolved from an inpatient to an outpatient process when low molecular weight heparin (LMWH) became available for subcutaneous administration at home, without the need for laboratory monitoring, thus obviating the need for hospitalization [9-12].

Although there continues to be a lack of awareness, mounting evidence [2,3,6,11,13-35] strongly suggests that the discontinuation of warfarin is not necessary for minor oral surgery procedures. There continues to be a widespread belief among patients, dentists, and physicians that oral anticoagulation and/or antiplatelet drugs must be held prior to dental extractions. The scientific literaturedoes not support the routine discontinuation of oral anticoagulation therapy for patients undergoing minor oral surgery procedures.

This preliminary study investigates the feasibility of utilizing a Point-of-Care In-Office INR Monitoring Device in the every day practice of oral and maxillofacial surgery and describes aninnovating protocol (Figure 1) that is safe, simple and efficient for both the surgeon and patient.

\section{Risk Stratification}

Patients are stratified $[3,26,36]$ into one of three risk categories for thromboembolism according to their indication for anticoagulant therapy.

- Low risk: 1-year risk of arterial embolism less than $5 \%$, or 1 -month of venous thromboembolism greater than $2 \%$;

- Moderate risk: 1-year risk of arterial embolism 5\% $10 \%$, or 1 -month risk of venous thromboembolism $2 \%$ to $10 \%$;

- High risk: 1-year risk of arterial embolism greater than $10 \%$, or 1 -month risk of thromboembolism greater than $10 \%$.

If the annual risk for thromboembolism is low, allowing for warfarin therapy to be withheld for 4 - 5 days prior to major surgery (without bridging) has been shown to pose very low risk to the patient $[3,26,36]$. On the contrary, withholding warfarin onmoderate to high-risk patients encompasses greater alarm for hazard with the potential of devastating sequela involving morbid disabilities or even death. Similarly, despite the evidence $[3,17,22,37,38]$ of platelet-altering medications not causing bleeding in minor oral surgery or dermatologic surgery procedures, dentists and physicians continue to hold aspirin, NSAIDS and clopidogrel (Plavix ${ }^{\circledR}$, Bristol-Myers Squibb/Sanofi Pharmaceuticals, New York, NY).

\section{PATIENTS AND METHODS}

A group of 61 consecutive adult patients on warfarin therapy were prospectively identified after referral to a oral and maxillofacial surgeon $(\mathrm{GH}, \mathrm{DB})$. The identified patients had to be on the oral anticoagulant warfarin on a chronic basis (greater than 3 months) and required "minor" oral and maxillofacial surgery. Patients were excluded from the study if "major" surgery was indicated. Dental extraction(s) (including impactions), alveoloplasty, bony tori/tuberosity reductions, placement of dental implants and minor oral mucosal or facial skin biopsies, excisions, or repair were considered "minor" surgery. Open treatment of facial trauma, orthognathic surgery, 


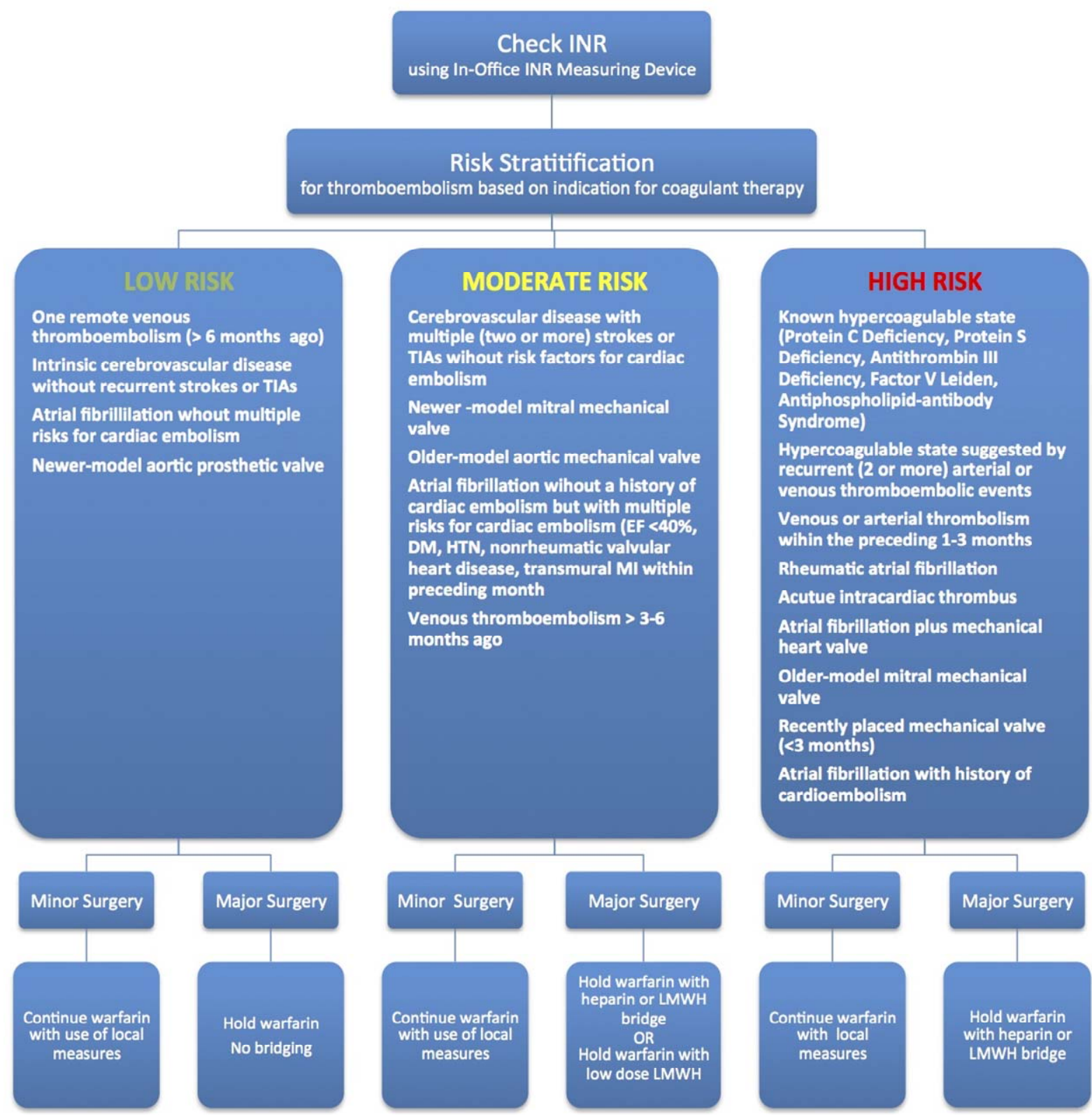

Figure 1. Algorithm for the perioperative management of the anticoagulated patient requiringoral surgery based on risk stratification, type of surgery, and utilization of In-Office INR Monitoring Device.

major pathology or reconstructive surgery requiring larger flaps or larger bone grafts, and invasive facial cosmetic procedures were considered "major" surgery. Before entering the study, patients were educated on possible risks and benefits related to the protocol and informed consent was obtained. The study received approval by the Good Shepherd Medical Center (Longview, TX) institutional review board.

Patients referred to office A (GH) were entered into the study Group A, in which warfarin and antiplatelet therapy was not discontinued. Group A was treated using a protocoloutlined in Figure 1, utilizing an In-Office
INR Monitoring Device. Patients referred to office B (DB) were entered into the control group. After consultation with the treating physician, warfarin therapy was withheld for 5 days prior to surgery and restarted on post-operative day 1. A preoperative INR was not obtained. Antiplatelet medications were also withheld for 7 days prior to surgery and restarted on post-operative day 1. Patients at too high of a risk to withhold warfarin were excluded from the study.

After a detailed history and physical, the patients in the study Group A, were treated using the protocol following the guidelines recommended by the American Col- 
lege of Chest Physicians [3]. First, patients were stratified into one of three risk categories: mild, moderate, or high risk based on the indications for anticoagulation. Next, indicated patient procedures were classified as "minor" or "major" surgery. At the consultation visit, a dental assistant obtained a small pin-prick blood sample which was analyzed by an INRatio ${ }^{\circledR}$ device (Inverness Medical Innovations, Waltham, MA). Patients with supra-therapeutic levels were warned and referred to their physician for adjustment. Medical clearance by the patient's treating physician was obtained via fax. On the day of surgery, the INR level was rechecked to assure safe and accurate levels, and surgery was performed if INR levels remained in a therapeutic range. Local anesthesia with a vasoconstrictor was used to aid in hemostasis. Atraumatic surgical technique (ex. Tooth sectioning) was used with careful handling of soft tissues to minimize trauma. Wounds were inspected for granulation tissue. Inflammatory/granulation tissue was meticulously removed from the surrounding periodontal tissues and periapical regions. Attempts were made to achieve primary wound closure when possible. Extraction sites were packed with oxidized regenerated cellulose (Surgicel ${ }^{\circledR}$, Johnson \& Johnson, New Brunswick, NJ), CollaPlug ${ }^{\circledR}$ (Colla-Tec, Inc, Plainsboro, NJ), or Gelfoam ${ }^{\circledR}$ (Upjohn, Kalamazoo, MI) and sutured with resorbable chromic gut. An Ellman ${ }^{\circledR}$ (Oceanside, NY) radiofrequency device was utilized to cauterize bleeding or oozing when performing biopsy or soft tissue procedures.

For a minimum of $30-60$ minutes, appropriate firm pressure was appliedby biting on $4 \times 4$ gauze in the recovery area under supervision of a dental assistant or RN. In order to meet the discharge criteria, the surgical site was reassessed for excessive oozing and appropriately charted prior to discharge. The importance of applying pressure was repetitively discussed at the consultation visit and in the recovery room. Patients and escorts were shown how to effectively fold and place gauze to insure hemostasis. Additionally, a discharge package including written postoperative instructions and extra gauze was handed to the patient prior to discharge. The patient was warned on the effects of supra-therapeutic INR levels. An attempt was made by the office staff to telephone all patients several hours after discharge. Post-operative visits were given on a PRN basis.

A "minor" intervention or complication was defined as a small hematoma or prolonged oozing that was easily controlled with local measures. A "major" complication was defined one requiring surgical intervention, admission to the hospital, severe loss of blood, need for transfusion or a thromboembolic event. After a 6 month period $(\mathrm{Oct} / 09-\mathrm{Mar} / 10)$ the medical records were used to collect the following information: age, gender, indication for anticoagulant therapy, INR level the day of surgery, concomitant antiplatelet medications, procedure(s) performed, and whether there were any complications or interventions.

Descriptive statistics, including mean, standard deviation, number of patients and percentages were determined. Differences in the treatment groups with respect to complications necessitating intervention were analyzed by the chi-squared test.

\section{RESULTS}

Of the 61 patients on chronic oral anticoagulation prospectively identified, 59 met the inclusion criteria to be included in the study (Table 1). The study Group A, in which the described protocol utilizing an In-Office INR Monitoring Device while maintaining oral anticoagulant therapy was made up of 30 patients. The control Group B was made up of 29 patients, all of whom were discontinued from their oral anticoagulant therapy was held. One patient in the study Group A was excluded from the study because of the necessity to undergo "major" surgery (pathological fracture of an atrophic mandible). A second patient in the control Group B was at too high of a risk to discontinue oral anticoagulation, thus excluded form the study.

In Group A (Table 2), there were 17 males and 13 females, ranging in age from 29 to 89 years with a mean age of 61.6 years. Indications for oral coagulation included atrial fibrillation ( $47 \%$ of patients, 14 total), mechanical heart valve (13\% of patients, 4 total), cerebrovascular disease ( $23 \%$ of patients, 7 total), history of venous thromboembolism ( $7 \%$ of patients, 2 total), or a known hypercoagulable state due to Lupus Anticoagulant Syndrome, Factor V Leiden, or Prothrombin mutation ( $20 \%$ of patients, 6 total). Seven patients $(23 \%)$ were stratified into low risk group, 13 patients $(43 \%)$ were represented in the moderate risk group, and 10 patients (33\%) were at high-risk (Figure 2). Thirty-five percent of the patients were on a concomitant antithrombolytic/antiplatelet medication. Eleven patients $(37 \%)$ were on aspirin of which two (7\%) were also taking aspirin and clopidogrel. INR levels ranged from 1.3 to 3.2 with a mean of $2.32( \pm 0.50$ standard deviation) (Figure 3). There were 6 patients $(20 \%)$ in the $1.0-1.99$ range, 21 patients $(70 \%)$ in the $2.0-2.99$ range, 3 patients $(10 \%)$ in the $3.0-3.99$ range, and there were no supratherapeutic values greater than 4.0. A discerning one of five patients in the group had subtherapeutic INR levels. After further questioning the low INRs were attributed to poor compliance or d rug and diet interactions, and not intentionally withholding warfarin.

Group A patients underwent a total of 131 separately codable "minor" procedures (Figure 4). A total of 108 teeth were extracted on 25 patients, ranging from 1 to 21 teeth (mean of $3.5 \pm 4.8$ ). Six of these patients underwent 
Table 1. Treatment group data comparison.

\begin{tabular}{|c|c|c|}
\hline & Group A (n = 30) (In-Office INR) & Group B $(n=29)($ Warfarin Withheld $)$ \\
\hline Gender (male/female) & $17 / 13$ & $20 / 9$ \\
\hline Mean age (range) & $61.6(29-89)$ & $58.9(37-79)$ \\
\hline \multicolumn{3}{|l|}{ Indications for anticoagulation } \\
\hline Atrial fibrillation (\%) & $14(47 \%)$ & $20(69 \%)$ \\
\hline Mechanical heart valve (\%) & $4(13 \%)$ & $3(10 \%)$ \\
\hline Cerebrovascular disease (\%) & $7(23 \%)$ & $4(14 \%)$ \\
\hline Venous thromboembolism (\%) & $2(7 \%)$ & $2(7 \%)$ \\
\hline Known hypercoagulable state (\%) & $6(20 \%)$ & 0 \\
\hline \multicolumn{3}{|l|}{ Risk Level } \\
\hline Low risk (\%) & $7(23 \%)$ & $14(48 \%)$ \\
\hline Moderate risk (\%) & $13(43 \%)$ & $14(48 \%)$ \\
\hline High risk (\%) & $10(33 \%)$ & $1(3 \%)$ \\
\hline \multicolumn{3}{|l|}{ Mean INR \pm SD (range) } \\
\hline $1.0-1.99(\%)$ & $2.36 \pm 0.47(1.3-3.2)$ & \\
\hline $2.0-2.99(\%)$ & $6(20 \%)$ & Not Measured \\
\hline $3.0-3.99(\%)$ & $21(70 \%)$ & \\
\hline$>4.0(\%)$ & $3(10 \%)$ & \\
\hline Antiplatelet medication withheld? & No & Yes \\
\hline Aspirin (\%) & $11(37 \%)$ & $4(14 \%)$ \\
\hline Clopidogrel (\%) & $2(7 \%)$ & $1(3 \%)$ \\
\hline Aspiring and Clopidogrel (\%) & $2(7 \%)$ & $1(3 \%)$ \\
\hline Procedures & 131 & 99 \\
\hline \#of patients requiring extractions & 25 & 24 \\
\hline Total extractions (range, mean) & $108(1-21,3.5)$ & $81(1-20,5.2)$ \\
\hline Impacted teeth & 8 & 0 \\
\hline Preprosthetic bone surgery & 19 & 10 \\
\hline Bone pathology/biopsy & 1 & 2 \\
\hline Intraoral soft tissue surgery/biopsy & 7 & 1 \\
\hline Facial skin surgery & 1 & 0 \\
\hline Incision and drainage & 1 & 0 \\
\hline Dental Implants & 2 & 4 \\
\hline Minor Bone Graft (Socket Preservation) & 0 & 1 \\
\hline Minor intervention(s) & 1 & 1 \\
\hline Major complications & None & None \\
\hline
\end{tabular}

removal of 8 impacted teeth with the remaining teeth coded as simple or surgical extractions. In the preprosthetic bone surgery group, seven patients underwent alveoloplasty, 1 patient underwent bilateral maxillary tuberosity reduction, and 1 patient underwent bilateral mandibular tori reduction. One patient underwent an anterior mandibular alveolectomyto facilitate the placement of two dental implants. Of the patients requiring surgery for pathology, one patient required simple enucleation of a mandibular radicular cyst. Six intraoral soft tissue biopsies with simple closure were performed on the tongue, gingival, lower lip, buccal mucosa $\times 2$, and floor of mouth. One patient underwent a $1.5 \mathrm{~cm}$ repair of facial squamous cell carcinoma wound defect. There was one patient in the infection category requiring an intraoral vestibular incision and drainage.

The control Group B consisted of 29 total patients with 20 males and 9 females, ranging in age from 39 to 79 years with a mean age of 58.7 years. Indications for oral coagulation included atrial fibrillation $(69 \%$ of patients, 20 total), mechanical heart valve ( $10 \%$ of patients, 3 total), cerebrovascular disease (14\% of patients, 4 total), and history of venous thromboembolism ( $7 \%$ of patients, 2 total). There were 14 low risk patients (48\%), 14 moderate risk patients (48\%), and one high risk patient (3\%). Seventeen percent of the patients were on a concomitant antithrombolytic/antiplatelet medication. Four patients were on aspirin, one patient was on clopi- 
Table 2. List of cases with indication for anticoagulation, thromboembolic risk level, concommitant antiplatelet medication(s), specific procedure(s) performed and any intervention or compication.

\begin{tabular}{|c|c|c|c|c|c|c|c|c|}
\hline \multirow{2}{*}{ CASE } & \multirow{2}{*}{ AGE } & \multirow{2}{*}{ SEX } & \multirow{2}{*}{ INDICATION } & \multirow{2}{*}{ RISK } & \multirow{2}{*}{ INR - } & \multirow{2}{*}{$\begin{array}{c}\text { ANTIPLATELET } \\
\text { MEDICATION }\end{array}$} & \multirow{2}{*}{ PROCEDURE(S) } & \multirow{2}{*}{$\begin{array}{l}\text { INTERVENTION OR } \\
\text { COMPLICATON }\end{array}$} \\
\hline & & & & & & & & \\
\hline 1 & 80 & $\mathrm{~F}$ & Atrial fibrillation & Low & 2.5 & - & Impaction + radicular cyst & \\
\hline 2 & 80 & M & Atrial fibrillation, CAD, HTN & Mod & 1.7 & ASA & 4 extractions & \\
\hline 3 & 62 & M & Atrial fibrillation, CAD, HTN & Mod & 2.6 & ASA, Clopidogrel & 9 extractions + alveoloplasty & $\begin{array}{l}\text { Prolonged oozing from } \\
\text { "liver clot"; cleaned out } \\
\text { and repacked }\end{array}$ \\
\hline 4 & 81 & M & Atrial fibrillation & Low & 1.5 & ASA & $\begin{array}{c}7 \text { extractions, bilateral maxillary } \\
\text { tuberosities }\end{array}$ & \\
\hline 5 & 56 & M & Atrial fibrillation & Low & 2.4 & - & 3 extractions + Gingival biopsy & \\
\hline 6 & 54 & M & $\begin{array}{c}\text { Mechanical aortic \& mitral } \\
\text { valves }\end{array}$ & High & 2 & - & $\begin{array}{c}\text { Impaction, } 12 \text { extractions, } \\
\text { alveoloplasty }\end{array}$ & \\
\hline 7 & 68 & M & CVA & Mod & 1.7 & - & $\begin{array}{l}13 \text { extractions, alveoloplasty, } \\
\text { tongue biopsy }\end{array}$ & \\
\hline 8 & 85 & $\mathrm{~F}$ & CVA & Mod & 2.3 & ASA & 1 extraction & \\
\hline 9 & 82 & M & Atrial fibrillation & Low & 2.5 & ASA & 4 extractions + alveoloplasty & \\
\hline 10 & 38 & $\mathrm{~F}$ & Prothrombin mutation & High & 2.1 & - & 4 impactions & \\
\hline 11 & 75 & M & Atrial fibrillation & Low & 2.8 & - & 2 extractions & \\
\hline 12 & 28 & $\mathrm{~F}$ & Factor V Leiden & High & 2.5 & - & 1 extraction & \\
\hline 13 & 29 & $\mathrm{~F}$ & Factor V Leiden & High & 2.3 & - & 1 extraction & \\
\hline 14 & 63 & M & Atrial fibrillation, $\mathrm{CHF}$ & Mod & 1.8 & ASA & 1 extraction & \\
\hline 15 & 63 & $\mathrm{~F}$ & Mechanical mitral valve & High & 3.1 & - & $\begin{array}{l}\text { Repair of facial skin } \\
\text { cancer }-1.5 \mathrm{~cm}\end{array}$ & \\
\hline 16 & 86 & M & Atrial fibrillation & Low & 2.9 & - & 1 extraction & \\
\hline 17 & 63 & $\mathrm{~F}$ & Atrial fibrillation & Low & 2.3 & - & $\begin{array}{c}21 \text { extractions, alveoloplasty, } \\
\text { mandibular tori }\end{array}$ & \\
\hline 18 & 84 & M & Atrial fibrillation, CAD, HTN & Mod & 2.1 & ASA & 1 extraction & \\
\hline 19 & 66 & M & $\begin{array}{c}\text { Atrial fibrillation, CAD, HTN, } \\
\text { CVA }\end{array}$ & High & 2.9 & ASA & Alveolectomy, 2 dental implants & \\
\hline 20 & 49 & $\mathrm{~F}$ & $\begin{array}{l}\text { Lupus Anticoagulant } \\
\text { Syndrome, DVT }\end{array}$ & High & 3.2 & - & 1 extraction & \\
\hline 21 & 45 & $\mathrm{~F}$ & Mechanical mitral valve & Mod & 2.5 & - & Lower lip and FOM biopsies & \\
\hline 22 & 51 & M & CVA & Mod & 2.7 & ASA & 2 mucosa biopsies & \\
\hline 23 & 35 & M & $\begin{array}{l}\text { Lupus Anticoagulant } \\
\text { Syndrome }\end{array}$ & High & 2.3 & - & $\begin{array}{c}4 \text { extractions, alveoplasty, ves- } \\
\text { tibular I \& D }\end{array}$ & \\
\hline 24 & 39 & $\mathrm{~F}$ & $\begin{array}{l}\text { Lupus Anticoagulant } \\
\text { Syndrome }\end{array}$ & High & 2.4 & - & 2 impactions, 2 extractions & \\
\hline 25 & 61 & M & Atrial fibrillation, CAD, HTN & Mod & 2.4 & ASA, Clopidogrel & 6 extractions & \\
\hline 26 & 49 & $\mathrm{~F}$ & DVT & Mod & 1.8 & ASA & 1 extraction & \\
\hline 27 & 55 & M & Mechanical aortic valve & High & 3.1 & - & $\begin{array}{l}\text { Referred from the ER for un- } \\
\text { controllable bleeding from a } \\
\text { periodontically involved tooth. } \\
\text { Treated by extraction, removing } \\
\text { of excess granulation tissue, and } \\
\text { bone wax to stop a small } \\
\text { mandibular arterial "pumper" }\end{array}$ & \\
\hline 28 & 58 & M & CVA & Mod & 2.6 & - & Tongue biopsy & \\
\hline 29 & 89 & $\mathrm{~F}$ & Atrial Fibrillation, CAD, CVA & Mod & 1.3 & - & 2 extractions & \\
\hline 30 & 59 & $\mathrm{~F}$ & CAD, HTN, CVA & Mod & 2.4 & - & 2 extractions & \\
\hline
\end{tabular}

dogrel, and one patient was on both aspirin and clopidogrel.
A total of 99 separately codable "minor" procedures were performed on the 29 patients in the control group. 


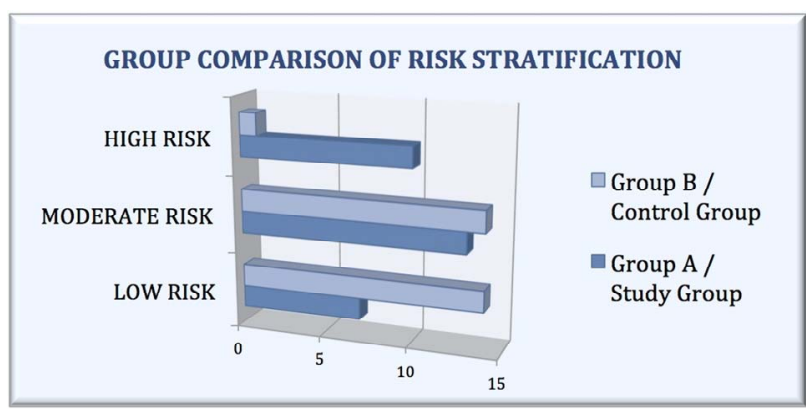

Figure 2. Chart comparing the 2 patient groups by risk stratification for thromboembolice events.

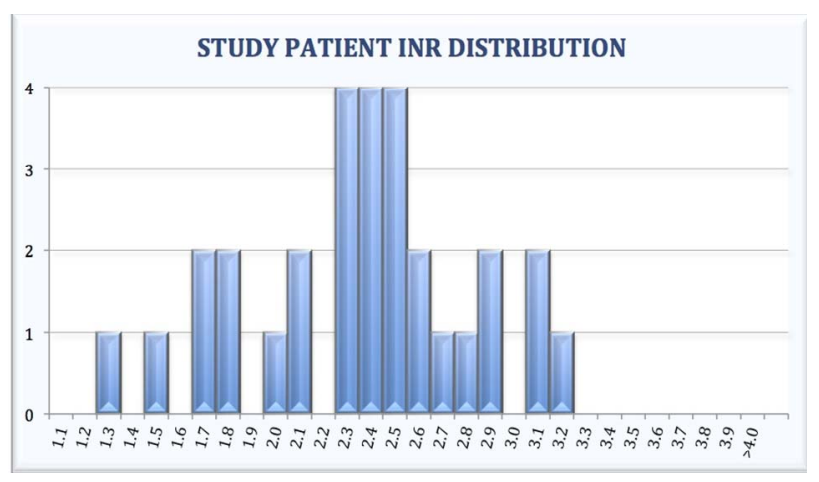

Figure 3. Study population INR level distribution day of procedure.

Eighty-one teeth were extracted on 24 patients, ranging from 1 to 20 teeth (mean of $5.2 \pm 4.5$ ) with no impactions. Preprosthetic bone surgery was performed on 3 patients requiring alveoloplasty, one of which also requiring bilateral mandibular tori reduction. Four dental implants were placed in 2 patients. Pathology procedures were performed on two patients requiring simple curetage/enucleation of minor cysts associated with the roots of teeth and one patient undergoing a soft tissue biopsy of the soft palate. A single patient opted for socket preservation with a bone graft for future implant placement.

Statistically, there was no significant difference with respect to complications between the 2 groups in the trial. There were no "major" complications in either the study or control groups, and each group had a single patient that required a "minor" intervention. The single Group A patient required intervention $(1 / 30 ; 3 \%)$ after returning to the office on post-operative day 2 with mild oozing after undergoing 9 extractions and alveoloplasty with an INR of 2.6 while taking aspirin and clopidogrel. This patient was easily managed by removing the "liver clot" and repacking oxidized regenerated cellulose, secured with a horizontal mattress suture. The single Group B patient $(1 / 29 ; 3 \%)$ requiring intervention also had persistent oozing on post-operative day 2 easily managed by packing ActCel ${ }^{\mathrm{TM}}$ hemostatic gauze. There were no after hour telephone calls to the oral surgeon on call or any emer-

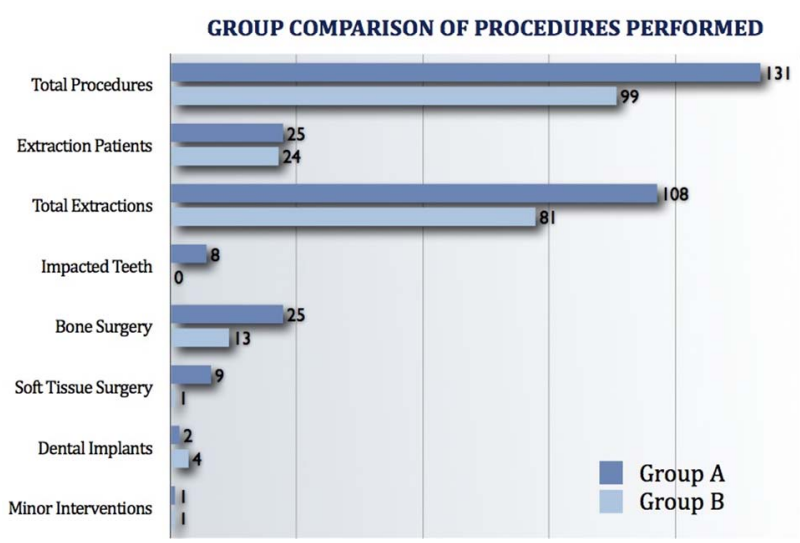

Figure 4. Chart comparing the number and type of procudures preformed in the 2 patient groups.

gency room visits related to bleeding or oozing of blood.

\section{DISCUSSION}

\subsection{Home Use of Self-Monitoring Devices}

The oral and maxillofacial surgeon is frequently required to treat patients who are receiving oral anticoagulant treatment. Warfarin is the most widely used anticoagulant in the North America with an estimated 4 million patients in the United States [18,36]. However, it requires careful laboratory supervision to prevent complications [39] because of several factors including individual response, diet, and concomitant medications that can interfere with the drug's effectiveness and safety [40]. The most common indications for warfarin therapy is in patients with mechanical heart valves, thromboembolic complications of atrial fibrillation, deep venous thrombosis, total hip arthroplasty, a history of pulmonary emboli, and to prevent recurrent myocardial infarctions and transient ischemic attacks.

Patients taking warfarin require frequent blood tests to maintain a safe and effective level of anticoagulation. A recent study demonstrated that patients are burdened with significant costs in time and travel expenses to the anticoagulation clinic [41].

PT/INR self-monitoring devices are Point-of-Care (POC) devices used to monitor blood-clotting rates by patients in the home similar to the use of glucometers by diabetic patients (Figure 5). Home PT/INR monitoring conveniently permits more frequent self-monitoring and management of anticoagulant therapy with warfarin. The ultimate goal is to maintain anticoagulation within a therapeutic INR (International Normalized Ratio) range to prevent hemorrhagic or thromboembolic events. A few examples of self-monitoring systems include the INRatio $^{\circledR}$ (Inverness Medical Innovations, Waltham, MA), Coaguchek $^{\circledR}$ (Roche Diagnostics, Basel, Switzerland), AvoSure $^{\mathrm{TM}}$ (Beckman Coulter,Fullerton, CA), Protime ${ }^{\mathrm{B}}$ 


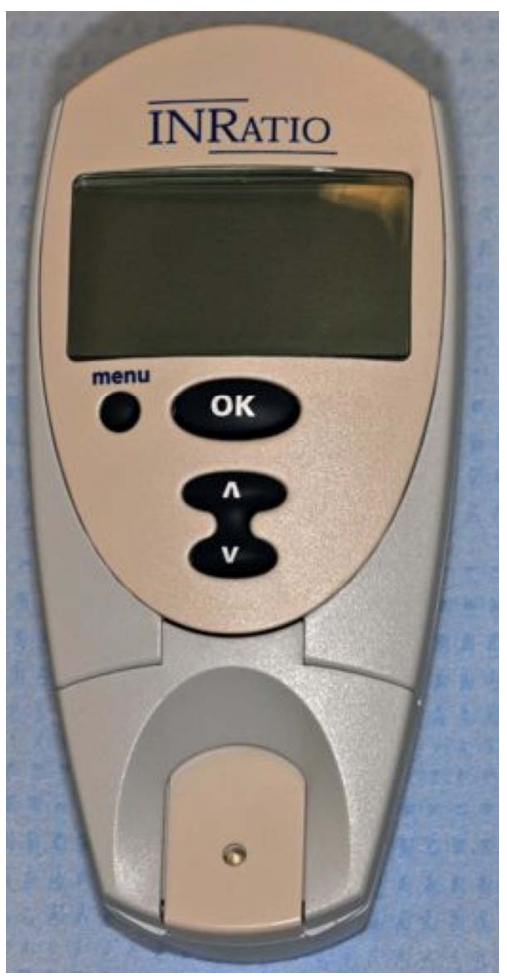

Figure 5. Medical office use of SelfMonitoring Devices.

Microcoagulation System and HEMOCHRON ${ }^{\circledR}$ (International Technidyne Corp., Edison, New Jersey). Several controlled studies in the literature assessing the quality of PT/INR self-monitoring systems effectiveness in maintaining a therapeutic level of anticoagulation [42-49].

Point-of-Care INR Monitoring Systems are composed of a monitor, disposable plastic reagent cartridges, and a finger stick blood collection kit. Devices usually store between 30 - 40 of the most recent test results, which are dated and stamped. With a simple finger stick to obtain a drop of blood, followed by insertion of the disposable cartridge into the device, the patient's results are obtained in minutes without the risk of waiting and transportation of samples or time delayed lab results. Devices are typically cost in the range of $\$ 1000$ to $\$ 2000$, however patients may acquire a monitor via third party medical insurance benefits.

Utilizing a Point-of-Care INR Monitoring Device in the oral and maxillofacial surgery office can facilitate the fast, easy and efficient care of anticoagulated patients. An In-Office INR Monitoring Device gives the oral surgeon the ability to provide a convenient, accelerated and concise service to the patient. INR levels are instantly obtained allowing for immediate decisions and less squandered time spent on patient counseling, direct communication with the treating physician, and inconvenience of coordinating surgery appointments with patients visits to the anticoagulation lab. Undesirable pro- longed lapses of sub-therapeutic warfarin blood levels as a consequence of patients withholding their medication for an extended period of time can be circumvented. Unreliable, misleading discrepancies in INR levels because the patient visited the anticoagulation lab several days prior to the surgery appointment can also be averted. Instant INR results obviate the necessity for deferring treatment so patients with severe pain or infections can be treated immediately on the day of consultation.

\subsection{Literature Review}

In-Office INR Monitoring Devices enable optimization of the treatment of patients under oral anticoagulation therapy prior to routine oral surgery procedures. In a study evaluation 298 dental surgery patients, Kruse-Loesler et al. [49] determined that In-Office PT/INR testing device values were clinically acceptable compared to traditional laboratory values. In 2008, Brennan et al. [25] published an article discussing the utility of an In-Office PT/INR testing device in a hospital-based dental practice.

The practice of interrupting oral anticoagulant or vitamin $\mathrm{K}$ antagonist therapy for dental procedures has been seriously questioned. The scientific literature $[2$, $3,6,11,13-35]$ does not support the routine discontinuation of oral anticoagulation therapy for patients undergoing minor oral surgery procedures.

In 1983 Bailey and Fordyce reported their experience with anticoagulated patients requiring extractions concluded that discontinuing anticoagulant therapy is not justified when in therapeutic range. In 1998, Wahl [50] published a case review of dental surgery in patients on anticoagulant therapy with 2014 procedures, including full mouth extractions and alveoloplasties performed on 774 patients. The results showed adequate hemostasis can be achieved with local measures. There was a $1.3 \%$ risk for severe bleedingwas linked to the majority of patients havingsupra-therapeutic intra- or post-operative anticoagulation levels. In 2000 Wahl [24] later published a review in JADA titled "Myths of dental surgery in patients receiving anticoagulant therapy", in which he wrote: "It is time to stop interrupting warfarin therapy for dental surgery".

The most recent meta-analysis of randomized controlled trials compared the effects of continuing the regular dose of warfarin therapy with the effects of discontinuing or modifying the dose on the incidence of bleeding in patients undergoing minor dental procedures [18]. With five trials (total of 553 patients) meeting the inclusion criteria the review concluded that continuing the regular dose of warfarin does not confer any increase of bleeding. With respect to warfarin patients that also take concomitant antiplatelet drugs, a Japanese study [22] failed to identify any significant differences between war- 
farin monotherapy and combination therapy. A retrospective analysis [51] published in 2007 concluded that use of LMWH bridge therapy is often unnecessary; contributing to inappropriate drug use associated with avoidable drug costs of more that $\$ 600$ per extraction.

\subsection{Fear of Lawsuit}

Despite growing evidence, most patients and care providers continue to discontinue warfarin. Even if practicing caregivers accept that there is a low risk of bleeding with not discontinuing warfarin, they may feel the need to interrupt therapy for fear of lawsuit after postoperative complications. Any fear should be redirected towardsa potentially devastating thromboembolic event after a minor oral surgery procedure.

\subsection{What Is the Acceptable INR for Minor Oral Surgery Procedures}

The use of variable "safe INR levels" for minor surgery continues to lack of full consensus $[5,6,27,31,48]$. The optimal INR value for minor oral surgery procedures is between 2.0 and 3.0. Procedures may safely be done while maintaining an INR value level of 3.0 to 4.0 as long as local hemostatic measures are taken. Surgery is contraindicated with an INR value of greater than 5.0.

\subsection{Partial Correction}

As Ward [6] points out, there are no studies to date involving significant numbers of moderate to high-risk procedures in our specialty. Unlike minor dentoalveolar or skin procedures (ex. dental extractions, dental implants, biopsies), invasive oral and maxillofacial procedures (ex. trauma surgery, facial cosmetic surgery, TMJ surgery) pose a greater risk of hemorrhagic complications requiring withholding of warfarin on low risk patients or use of heparin bridge on high-risk patients. As described in feasibility study [52] evaluating 100 high-risk patients that underwent more invasive surgery (ex. lap cholecystectomy, hip/knee replacement, major GI/vascular surgery, cardiothoracic surgery), moderate-intensity anticoagulation therapy with warfarin (INR of $1.5-2.0$ ) appeared as a safe and reasonable alternative method. There is little to no evidence supporting this method for more invasive OMS procedures (facial trauma, TMJ, sleep apnea, cosmetic surgery). This technique of partial correction was advocated in the management of dental extractions in a study using rabbits in 1992 [53].

\subsection{The Use of Local Measures}

It is imperative to use include local measures when treating anticoagulated patients. Minimally, a hemostatic matrix such as oxycellulose, absorbable gelatin, or col- lagen with sutures should be used [2,22,30]. When performing procedures on patients without discontinuing warfarin, special care of attention must be paid to the surrounding tissues. As previously described [22], patients with severe periodontal disease or periapical pathology have increased local inflammation and are at an increased risk of bleeding. It is very important to remove all granulation tissue from the extraction site. To set optimal conditions it has been suggested that patients visit the hygienist 1 week prior to surgery for plaque and calculus removal [23].

Several other modalities have been effectively utilized for hemostasis after minor oral surgery procedures. Electrocautery may be used if hemostasis cannot be achieved in inflamed, highly vascular tissue or a blood vessel in encountered [31]. Rinsing with an antifibrinolytic agent like tranexamic acid or $\varepsilon$-aminocaproic acid (5\%) 4 times a day for 2 minutes for 2 to 5 days postoperatively. $[2,3,14,28,30,54]$ Fibrin glue $[14,30,55,56]$ and platelet rich plasma (PRP) [15] has also been shown to be successful. To minimize risk of bleeding some surgeons have advocated treated patients over more than one visit by treating an arch or a quadrant per visit [57].

The vast majority of minor oral surgery procedures are undertaken outside the hospital, in an office setting. In order to meet the discharge criteria, adequate hemostasis, with only minimal oozing is achieved prior to discharge. Consequently, any post-operative bleeding or excessive oozing will occur at home that may generate concern and anxiety. It is imperative to stress the importance of gauze pressure at the consultation visit and then repeat the instructions prior to discharge to both the patient and escort. Patients are advised on what is considered excessive warranting medical attention.

\subsection{Antithrombotic Therapy}

As minor oral surgery or dermatological procedures are typically associated with relatively little blood loss, a key question arises about the safety of continuing antithrombotic agents. Abundant evidence [3,17,22,37,38] affirms that platelet-altering medications (Aspirin, NSAIDS, clopidogrel) do not increase the risk of bleeding in minor oral surgery or dermatologic surgery. American College of Chest Physicians Evidence-Based Clinical Practice Guidelines recommend continuing aspirin around the time of minor oral surgery or dermatologic procedure (Grade 1C evidence) [3].

\subsection{Reimbursement}

The average upfront cost of a device is roughly $\$ 1500$. The Centers for Medicare \& Medicaid Services (CMS) and the majority of third party insurance companies will reimburse the healthcare provider when there is proper 
medical necessity submitted as an ICD-9 (diagnosis) code on a claim. The Prothrombin Time procedure code (CPT 85610) can be used with the QW modifier, since it is a CLIA-waived test. A provider may not bill an additional code for obtaining a blood sample. The test result must be documented in the patient's chart. The oral surgeon must have a CLIA certificate when performing any type of testing including a blood glucose test on a diabetic or a pregnancy test prior to anesthesia induction.

\subsection{Conclusion}

The use of an In-Office INR Monitoring Device transforms the inconvenient and often cumbersome and timeconsuming task of managing anticoagulated patients. Less time is spent on patient education and scheduling surgery no longer needs to be coordinated with a trip to the anticoagulation lab. The process is simple, safe, quick and effective.

When practicing medicine or dentistry, it is important to remember to evaluate each patient and their co-morbid factors independently without the use of a single across-the-board recommendation. Todd (1) advocates that flexibility is required and in some cases modification of anticoagulation regimen is beneficial for performance of the procedure and the postoperative course. Oral and maxillofacial surgeons should collaborate and communicate with referring dentists and appropriately consult and with the primary care physician or medical specialist as indicated. Our office faxes a simple, yet adequately detailed medical clearance form to the anticoagulation managing physician. A written quality control policy has been implemented to ensure that consultations are received by the physician and timely returned to our office prior the patient's scheduled surgery visit. In my personal experience, greater than $90 \%$ of physicians routinely discontinue both perioperative warfarin and antiplatelet medications which has since prompted me to add a short note to the medial clearance form stating that continuing these medications is acceptable from a surgical standpoint as long as the patient is in the therapeutic range.

Recently, I received a phone call in the evening from an ER physician who was having difficulty obtaining hemostasis after a dental extraction performed by a local dentist earlier in the day. The dentist failed to get a good history and didn't recognize that the patient had a history of chronic anemia, coronary artery disease with a previous myocardial infarction and was taking warfarin. In the ER, an INR was ordered and was found to be 3.1. My examination revealed a perfuse bleed from what looked like a simple extraction. Local measures including extended gauze pressure, injection of local anesthesia with a vasoconstrictor, packing the extraction site with oxidized regenerated cellulose impregnated with $\varepsilon$-aminocaproic acid, placement of a suture, and the use of topical bovine-derived gelatin matrix and thrombin (FloSeal Matrix, Baxter, Deerfield, IL) all failed to control the bleeding (likely due to clotting factor deficiency). The patient's hemoglobin dropped from a baseline of 13.0 to 6.9 , and the patient started exhibiting signs of severe blood loss including pallor, altered mental status, tachycardia with increased PVCs. Immediate PRBC and FFP transfusions were ordered along with hematology and cardiology consultations. The patient thankfully responded well to the transfusion and reversal of anticoagulation and was discharged without any permanent sequel after a 5-day hospital course.

This case is a classic example of why oral and maxillofacial surgeons must constantly be thorough and pay attention to detail while treating each patient on an individual basis, as previously advised by Assael's [58] Journaleditorial discussing hemostasis. New tools have permitted oral and maxillofacial surgeons to make exceptional contributions to the overall health of patients. I believe that the In-Office INR Monitoring Device is another useful another tool to add to your practice.

\section{CONCLUSION}

In conclusion, for most patients undergoing minor oral surgery procedures, the devastating sequelae of withholding anticoagulant therapy greatly outweighs the risk of prolonged bleeding if anticoagulant therapy is continued. Continuing the regular dose of warfarin therapy does not seem to confer an increased risk of bleeding. This preliminary investigation has demonstrated the convenience, simplicity, efficiency, low cost, and safety associated with the utilization of an In-Office Point-ofCare INR Monitoring Device when managing every day oral and maxillofacial surgery patients on warfarin. Given the small samples size, further investigation with larger randomized, controlled studies may further validate the incorporation of a Point-of Care INR Monitoring Device in every contemporary oral and maxillofacial surgery office.

\section{REFERENCES}

[1] Todd, D.W. (2005) Evidence to support an individualized approach to modification of oral anticoagulant therapy for ambulatory oral surgery. Journal of Oral and Maxillofacial Surgery, 63, 536. doi:10.1016/j.joms.2004.12.008

[2] Beirne, O.R. (2005) Evidence to continue oral anticoagulant therapy for ambulatory oral surgery. Journal of Oral and Maxillofacial Surgery, 63, 540. doi:10.1016/j.joms.2004.12.009

[3] Douketis, J.D., Berger, P.B., Dunn, A.S., et al. (2008) The perioperative management of antithrombotic therapy: American College of Chest Physicians Evidence-Based Clinical Practice Guidelines (8th Edition). Chest, 133, 299S. 
[4] Lim, W., Wang, M., Crowther, M. and Douketis, J. (2007) The management of anticoagulated patients requiring dental extraction: A cross-sectional survery of oral and maxillofacial surgeons and hematologists. Journal of Thrombosis and Haemostasis, 5, 2157. doi:10.1111/j.1538-7836.2007.02700.x

[5] Troulis, M.J., Head, T.W. and Leclerc, J.R. (1998) Dental extractions in patients on an oral anticoagulant: A survey of practices in North America. Journal of Oral and Maxillofacial Surgery, 56, 914. doi:10.1016/S0278-2391(98)90647-4

[6] Ward, B.B. and Smith, M.H. (2007) Dentoalveolar procedures for the anticoagulated patient: Literature recommendations versus current practice. Journal of Oral and Maxillofacial Surgery, 65, 1454. doi:10.1016/j.joms.2007.03.003

[7] Mehra, P., Cottrell, D.A., Bestgen, S.C., et al. (2000) Management of heparin therapy in the high-risk, chronically anticoagulated, oral surgery patient: A review and proposed nomogram. Journal of Oral and Maxillofacial Surgery, 58, 198. doi:10.1016/S0278-2391(00)90339-2

[8] Aframian, D.J., Lalla, R.V. and Peterson, D.E. (2007) Management of dental patients taking common hemostatsis-altering medications. Oral Surgery, Oral Medicine, Oral Pathology, Oral Radiology, 103, s45.

[9] Douketis, J.D., Johnson, J.A. and Turpie, A.G. (2004) Low-Molecular-Weight Heparin as bridging anticoagulation during interruption of warfarin: Assessment of a standardized periprocedural anticoagulation regimen. Archives of Internal Medicine, 164, 1319. doi:10.1001/archinte.164.12.1319

[10] Todd, D.W. and Roman, A. (2001) Outpatient use of low-molecular weight heparin in an anticoagulated patient requiring oral surgery: Case report. Journal of Oral and Maxillofacial Surgery, 59, 1090. doi:10.1053/joms.2001.25855

[11] Jaffer, A.K., Ahmed, M., Brotman, D.J., et al. (2005) Low-molecular-weight heparins as periprocedural anticoagulation for patients on long-term warfarin therapy: A standardized bridging therapy protocol. Journal of Thrombosis and Thrombolysis, 20, 11. doi:10.1007/s11239-005-3120-9

[12] Bajkin, B.V., Popovic, S.L. and Selakovic, S.D. (2009) Randomized, prospective trial comparing bridging therapy using low-molecular-weight heparin with maintenance of oral anticoagulation during extraction of teeth. Journal of Oral and Maxillofacial Surgery, 67, 990. doi:10.1016/j.joms.2008.12.027

[13] Bailey, B.M. and Fordyce, A.M. (1983) Complications of dental extractions in patients receiving warfarin anticoagulant therapy. A controlled clinical trial. British Dental Journal, 155, 308. doi:10.1038/sj.bdj.4805221

[14] Carter, G., Goss, A., Lloyd, J. and Tocchetti, R. (2003) Tranexamic acid mouthwash versus autologous fibrin glue in patients taking warfarin undergoing dental extractions: A randomized prospective clinical study. Journal of Oral and Maxillofacial Surgery, 61, 1432. doi:10.1016/j.joms.2003.01.001

[15] Valle, A.D., Sammartino, G., Marenzi, G., et al. (2003)
Prevention of post-operative bleeding in patients undergoing oral surgery: Use of platelet-rich plasma gel. Journal of Oral and Maxillofacial Surgery, 61, 1275. doi:10.1016/S0278-2391(03)00727-4

[16] Souto, J.C., Oliver, A., Zuazu-Juasoro, I., et al. (1996) Oral surgery in anticoagulated patients without reducing the dose of oral anticoagulant: A prospective randomized study. Journal of Oral and Maxillofacial Surgery, 54, 27. doi:10.1016/S0278-2391(96)90297-9

[17] Pototski, M. and Amenábar, J.M. (2007) Dental management of patients receiving anticoagulation or antiplatelet treatment. Journal of Oral Science, 49, 253-258. doi:10.2334/josnusd.49.253

[18] Nematullah, A., Alabousi, A., Blanas, N., Douketis, J.D. and Sutherland, S.E. (2009) Dental surgery for patients on anticoagulant therapy with warfarin: A systematic review and meta-analysis. Journal of the Canadian Dental Association, 75, 41.

[19] Beirne, O.R. and Koehler, J.R. (1996) Surgical management of patients on warfarin sodium. Journal of Oral and Maxillofacial Surgery, 54, 1115. doi:10.1016/S0278-2391(96)90172-X

[20] Campbell, J.H., Alvarado, F. and Murray, R.A. (2000) Anticoagulation and minor oral surgery: Should the anticoagulation regimen be altered? Journal of Oral and Maxillofacial Surgery, 58, 131.

[21] Jeske, A.H. and Suchko, G.D. (2003) Lack of a scientific basis for routine discontinuation of oral anticoagulation therapy before dental treatment. The Journal of the American Dental Association, 134, 1492. doi:10.1016/S0278-2391(00)90324-0

[22] Morimoto, Y., Niwa, H. and Minematsu, K. (2008) Hemostatic management of tooth extractions in patients on oral antithrombotic therapy. Journal of Oral and Maxillofacial Surgery, 66, 51. doi:10.1016/j.joms.2007.06.655

[23] Ferrieri, G.B., Castiglioni, S., Carmagnola, D., Cargnel, M., Strohmenger, L. and Abati, S. (2007) Oral surgery in patients on anticoagulant treatment without therapy interruption. Journal of Oral and Maxillofacial Surgery, 65, 1149. doi:10.1016/j.joms.2006.11.015

[24] Wahl, M.J. (2000) Myths of dental surgery in patients receiving anticoagulant therapy. The Journal of the American Dental Association, 131, 77.

[25] Brennan, M.T., Hong, C., Furney, S.L., Fox, P.C., et al. (2008) Utility of an International Normalized Ratio testing device in a hospital-based dental practice. The Journal of the American Dental Association, 139, 697.

[26] Hirsh, J., Fuser, V., Ansell, J., et al. (2003) American Heart Association/American College of Cardiology Foundation Guide to warfarin therapy. Circulation, 107, 1692. doi:10.1161/01.CIR.0000063575.17904.4E

[27] Blinder, D., Manor, Y., Martinowitz, U. and Taicher, S. (2001) Dental extractions in patients maintained on oral anticoagulant therapy: Comparison of INR value with occurrence of postoperative bleeding. International Journal of Oral and Maxillofacial Surgery, 30, 518. doi:10.1054/ijom.2001.0172 
[28] Zanon, E., Martinelli, F., Bacce, C., et al. (2003) Safety of dental extraction among consecutive patients on oral anticoagulant treatment managed using a specific dental management protocol. Blood Coagulation \& Fibrinolysis, 14, 27. doi:10.1097/00001721-200301000-00006

[29] Evans, I.L., Sayer, M.S., Gibbons, A.J., et al. (2002) Can warfarin be continued during dental extractions? Results of a randomized controlled trial. British Journal of Oral and Maxillofacial Surgery, 40, 248. doi:10.1054/bjom.2001.0773

[30] Blinder, D., Manor, Y., Martinowitz, U., Taicher, S. and Hashomer, T. (1999) Dental extractions in patients maintained on continued oral anticoagulant: Comparison of local hemostatic modalities. Oral Surgery, Oral Medicine, Oral Pathology, Oral Radiology, 88, 137. doi:10.1016/S1079-2104(99)70106-X

[31] Morimoto, Y., Niwa, H. and Minematsu, K. (2009) Hemostatic management for periodontal treatments in patients on oral antithrombotic therapy: A retrospective study. Oral Surgery, Oral Medicine, Oral Pathology, Oral Radiology, 108, 889. doi:10.1016/j.tripleo.2009.07.048

[32] Salam, S., Yusuf, H. and Milosevic, A. (2007) Bleeding after dental extractions in patients taking warfarin. British Journal of Oral and Maxillofacial Surgery, 45, 463. doi:10.1016/j.bjoms.2006.12.004

[33] Sacco, R., Sacco, M., Carpenedo, M. and Moia, M. (2006) Oral surgery in patients on oral anticoagulant therapy: A randomized comparison of different INR targets. Journal of Thrombosis and Haemostasis, 4, 688. doi:10.1111/j.1538-7836.2006.01762.x

[34] Al-Mubarak, S., Rass, M.A., Alsuwyed, A., et al. (2006) Thromboembolic risk and bleeding in patients maintaining or stopping oral anticoagulant therapy during dental extraction. Journal of Thrombosis and Haemostasis, 4, 689. doi:10.1111/j.1538-7836.2006.01825.x

[35] Scully, C. and Wolff, A. (2002) Oral surgery in patients on anticoagulant therapy. Oral Surgery, Oral Medicine, Oral Pathology, Oral Radiology, 94, 57. doi:10.1067/moe.2002.123828

[36] Jaffer, A.K., Brontman, D.J. and Chukwumerije, N. (2003) When patients on warfarin need surgery. Cleveland Clinic Journal of Medicine, 70, 973-984. doi:10.3949/ccjm.70.11.973

[37] Billingsley, E.M. and Maloney, M.E. (1997) Intraoperative and postoperative bleeding problems in patients taking warfarin, aspirin, and nonsteroidal anti-inflammatory agents. Dermatologic Surgery, 23, 381-383. doi:10.1111/j.1524-4725.1997.tb00065.x

[38] Partridge, C.G., Campbell, J.H. and Alvarado, F. (2008) The effect of platelet-altering medications on bleeding from minor oral surgery procedures. Journal of Oral and Maxillofacial Surgery, 66, 93-97. doi:10.1016/j.joms.2005.11.055

[39] Wysowski, D.K., Nourjah, P. and Swartz, L. (2007) Bleeding complications with warfarin use: A prevalent adverse effect resulting in regulatory action. Archives of Internal Medicine, 167, 1414-1419.

doi:10.1001/archinte.167.13.1414
[40] Holbrook, A.M., Pereira, J.A., Labiris, R., McDonald, H., et al. (2005) Systematic overview of warfarin and its drug and food interactions. Archives of Internal Medicine, 165, 1095-1106. doi:10.1001/archinte.165.10.1095

[41] Jowett, S., Stirling, B., Isabelle, M., David, B., et al. (2008) A multinational investigation of time and traveling costs in attending anticoagulation clinics. Value in Health, 11, 207-212. doi:10.1111/j.1524-4733.2007.00253.x

[42] Heneghan, C., et al. (2006) Self-monitoring of oral anticoagulation: A systematic review and meta-analysis. The Lancet, 367, 404-411. doi:10.1016/S0140-6736(06)68139-7

[43] Christensen, T.D., Maegaard, M., Sørensen, H.T., Hjortdal, V.E. and Hasenkam, J.M. (2007) Self-versus conventional management of oral anticoagulant therapy: Effects on INR variability and coumarin dose in a randomized controlled trial. American Journal of Cardiovascular Drugs, 7, 191-197. doi:10.2165/00129784-200707030-00005

[44] Christensen, T.D., Maegaard, M., Sørensen, H.T., Hjortdal, V.E. and Hasenkam, J.M. (2006) Self-management versus conventional management of oral anticoagulant therapy: A randomized, controlled trial. European Journal of Internal Medicine, 17, 260-266. doi:10.1016/j.ejim.2005.11.021

[45] Christensen, T.D., Johnsen, S.P., Hjortdal, V.E. and Hasenkam, J.M. (2007) Self-management of oral anticoagulant therapy: A systematic review and meta-analysis. International Journal of Cardiology, 118, 54-61. doi:10.1016/j.ijcard.2006.06.018

[46] Kitchen, D.P., Kitchen, S., Jennings, I., Woods, T., et al. (2008) Quality assessment in point-of-care coagulation testing. Semin Thromb Hermost, 34, 647-653. doi:10.1055/s-0028-1104543

[47] Du Breuil, A.L. and Umland, E.M. (2007) Outpatient management of anticoagulation therapy. American Family Physician, 75, 1031-1042.

[48] Van den Besselaar, A.M. (2001) Accuracy, precision, and quality control for point-of-care testing of oral anticoagulation. Journal of Thrombosis and Thrombolysis, 12 , 35-40. doi:10.1023/A:1012734426811

[49] Kruse-Loesler, B., Kelker, M. and Kleinheinz, J. (2005) Comparison of laboratory and immediate diagnosis of coagulation for patients under oral anticoagulation therapy before dental surgery. Head \& Face Medicine, 1, 12. doi:10.1186/1746-160X-1-12

[50] Wahl, M.J. (1998) Dental surgery in anticoagulated patients. Archives of Internal Medicine, 158, 1610-1616. doi:10.1001/archinte.158.15.1610

[51] Pettinger, T.K. and Owens, C.T. (2007) Use of low-molecular-weight heparin during dental extractions in a medicaid population. Journal of Managed Care Pharmacy, 13, 53-58.

[52] Larson, B.J., Zumberg, M.S. and Kitchens, C.S. (2005) A feasibility study of continuing dose-reduced warfarin for invasive procedures in patients with high thromboembolic risk. Chest Journal, 127, 922-927. doi:10.1378/chest.127.3.922 
[53] DeClerck, D., Vinckier, F. and Vermylen, J. (1992) Influence of anticoagulation on blood loss following dental extraction. Journal of Dental Research, 71, 387-390. doi: $10.1177 / 00220345920710020701$

[54] Carter, G. and Goss, A. (2003) Transexamic acid mouthwash-A prospective randomized study of a 2-day regimen vs. 5-day regimen to prevent postoperative bleeding in anticoagulated patients requiring dental extractions. International Journal of Oral and Maxillofacial Surgery, 32, 504.

[55] Bodner, L., Weinstein, J.M. and Baumgarten, A.K. (1998) Efficacy of fibrin sealant in patients on various levels of oral anticoagulant undergoing oral surgery. Oral Surgery,
Oral Medicine, Oral Pathology, Oral Radiology, 86, 421.

[56] Halfpenny, W., Fraser, J.S. and Adlam, D.M. (2001) Comparison of 2 haemostatic agents for the prevention of postextraction hemorrhage in patients on anticoagulants. Oral Surgery, Oral Medicine, Oral Pathology, Oral Radiology, 92, 257.

[57] Malden, N.J., Santini, A., Mather, C.I. and Gardner, A. (2007) Minor oral surgery and interference with anticoagulation in patients taking warfarin: A retrospective study. British Journal of Oral and Maxillofacial Surgery, 45, 645.

[58] Assael, L.A. (2003) Hemostasis is a shared responsibility. Journal of Oral and Maxillofacial Surgery, 61,1377. 